\title{
Lung Physiological Variations in COVID-19 Patients and Inhalation Therapy Development for Remodeled Lungs
}

\author{
Jinxiang $\mathrm{Xi}^{1 *}$, Brendan Walfield ${ }^{1}$, Xiuhua April Si ${ }^{2}$, Alexander A. Bankier ${ }^{3,4}$ \\ ${ }^{1}$ Department of Biomedical Engineering, University of Massachusetts, Lowell, MA, United States. \\ ${ }^{2}$ Department of Aerospace, Industrial, and Mechanical Engineering, California Baptist University, Riverside, CA, United States. \\ ${ }^{3}$ Department of Radiology, University of Massachusetts Medical School, Worcester, MA, United States. \\ ${ }^{4}$ Department of Radiology, UMass Memorial Medical Center, Worcester, MA, United States.
}

Received 12 March 2021; Revised 09 May 2021; Accepted 24 June 2021; Published 01 September 2021

\begin{abstract}
In response to the unmet need for effective treatments for symptomatic patients, research efforts of inhaled therapy for COVID-19 patients have been pursued since the pandemic began. However, inhalation drug delivery to the lungs is sensitive to the lung anatomy and physiology, which can be significantly altered due to the viral infection. The ensued ventilation heterogeneity will change distribution and thus dosimetry of inhaled medications, rendering previous correlations concepts? of pulmonary drug delivery in healthy lungs less reliable. In this study, we first reviewed the recent developments of inhaled therapeutics and vaccines, as well as the latest knowledge of the lung structural variations documented by CT of COVID-19 patients' lungs. We then quantified the volume ratios of the poorly aerated lungs and non-aerated lungs in eight COVID-19 patients, which ranged 2-8\% and 0.5-3\%, respectively. The need to consider the diseased lung physiologies in estimating pulmonary delivery was emphasized. Diseased lung geometries with varying lesion sites and complexities were reconstructed using Statistical Shape Modeling (SSM). A new segmentation method was applied that could generate patient-specific lung geometries with an increased number of branching generations. The synergy of the CT-based lung segmentation and SSM-based airway variation showed promise for developing representative COVID-infected lung morphological models and investigating inhalation therapeutics in COVID-19 patients.
\end{abstract}

Keywords: COVID-19; Airway Remodeling; Inhalation Therapy; Inhaled Vaccine; Bronchial Constriction; Statistical Shape Modeling (SSM).

\section{Introduction}

The SARS CoV-2 virus has caused the worst pandemic worldwide in nearly a century, which up to date (June 6, 2021) has led to 173.01 million confirmed cases and 3.727 million deaths, according to WHO Coronavirus (COVID19) Dashboard [1]. The constant evolving variants of the SARS CoV-2 virus make the preventive and therapeutic efforts highly challenging, as evidenced by the outbreaks in Europe between October. 2020 and January 2021, and in south-east Asia from April 2021 till now [1]. Scientists all over the world are racing to find effective treatments and develop vaccines. The International Society of Aerosol Medicine (ISAM) deemed it an urgent need to rapidly develop inhaled therapies for COVID-19, which should consider the initial infection site, the disease progression route, and in particular the pulmonary alveoli where "cytokine storms" can cause a fatal cascade, including respiratory failure [2].

* Corresponding author: jinxiang_xi@uml.edu

doi) http://dx.doi.org/10.28991/SciMedJ-2021-0303-1

$>$ This is an open access article under the CC-BY license (https://creativecommons.org/licenses/by/4.0/).

(C) Authors retain all copyrights. 
In order to develop a dose-response relationship, it is critical to determine the actual dose that has been delivered to the diseased tissues. This dose should be quantified with regard to both quantity and distribution in the lung, as well as the dose variability among different subjects. Current pulmonary drug delivery suffers from drug waste in the mouth and throat and prevailing deposition in the conducting lungs, leaving relatively few aerosol particles reaching the alveoli. Lung structural remodeling and associated ventilation heterogeneity can alter particle dynamics and therefore impact the dosimetry and distribution of the inhaled medications. The lung physiological variation due to COVID-19 infections can be significant and the previous knowledge of pulmonary drug delivery in healthy lungs can be no longer reliable [3-6].

The following sections will present the recent developments of inhaled therapies for COVID-19 patients, the physiological variations in COVID-19 patient's lungs, the new need to intergrade these variations into the estimation of pulmonary dosimetry, and some latest developments in simulating lung structural remodeling and patient-specific lung reconstruction.

\section{Recent Developments in COVID-19 Inhaled Therapies}

The rationale for recent developments of inhaled therapeutics and vaccines over other routes is that coronavirus enters the human body predominantly through the respiratory tract, which is also the main site of viral replication, function deterioration, and potentially lethal cytokine storm. Dispensing drugs or vaccines directly to the diseased sites in the lung can elicit a fast and strong therapeutic outcome or mucosal immune response [7]. Due to the localized high doses on the target cells only, low systemic toxicity is also expected, thus minimizing adverse side effects associated with injection/oral use. For a given dose, inhalation therapy has been shown to have substantially higher efficacy than therapies administered through other routes [8]. For instance, compared to muscle injection of the albumin-modified vaccine, inhalation therapy elicited a 25 -fold boost of the memory $\mathrm{T}$ cells in the mouse lungs [9]. Inhalation therapeutics against the SARS-CoV-2 virus may allow future self-administered treatments by COVID-19 patients, reducing the need to go to the hospital, and thus reducing the chances of cross-transmission and ease the demands on medical facilities.

\subsection{Inhaled Treatments for Symptomatic Patients}

In response to the need for effective treatments for symptomatic patients, research efforts and clinical trials of inhaled therapy for COVID-19 patients have been actively undertaken since the start of the pandemic. Inhaled budesonide has been tested in the UK in the form of dry powders using a Turbuhaler in patients with mild symptoms, with the endpoint being COVID-related urgent care visits [10]. Ensifentrine, a dual inhibitor of the enzyme phosphodiesterase 3 and 4 (PDE3 and PDE4), which is often used as a bronchodilator and anti-inflammatory agent, was delivered using pMDI to evaluate its effectiveness in reducing mucous viscosity and improving mucociliary clearance [11]. High-concentration (160-200 ppm) nitric oxide delivered via facemask has been explored as rescue therapies to prevent coronavirus disease progression and hypoxic respiratory failure [12, 13]. Inhaled corticosteroids (ICS), which is a standard therapy for patients with chronic obstructive pulmonary disease (COPD), have been tested for their role in suppressing SARS-CoV-2 replication and reducing inflammation [14]. Despite positive results in cultured cells [14], reports of failed outcomes to decrease COVID-related mortality in patients with COPD or asthma have also been reported [15]. Unfractionated heparin (UFH) has anticoagulant, anti- inflammatory, and antiviral effects; nebulized delivery of UFH to lungs may prevent the entry of SARS-CoV-2 virus into respiratory cells and limit lung injury progression and vascular thrombosis [16]. Similarly, nebulization of IFN- $\alpha-2 b$ is being tested in China and France to treat viral COVID-induced pneumonia [17]. The suitability of Hydroxychloroquine (HCQ) as inhalable powders was tested by Albariqi et al. [18] using jet-milled techniques. The small powder size (1.7 um) and reasonably long shelf lifetime allow it to be used as a potential inhaled COVID-19 treatment option. Ferrer et al. evaluated the application of xylitol and grapefruit seed extract (GSE) as nasal sprays to reduce virus attachment to the nasal cells and thus as a preventive measure to curb viral infections not just for SARS-CoV-2 but also for other viral epidemics [19].

\subsection{Inhaled Vaccines}

There are 13 COVID-19 vaccines in use across the globe (Coronavirus Vaccine Tracker) and all of them are administered as shots to muscles of the upper arm [14]. An inhaled vaccine could be more effective than those injected given that the coronavirus enters the human body through the airways and delivered vaccines can induce a direct mucosal immune response. Considering the nature of being self-administration and non-injection, inhaled vaccines can also facilitate mass vaccination campaigns. As SARS-COV-2 binds human cells via spike protein receptors, antibodies, peptides, or hormones can be used to target these receptors [20, 21]. Neutralized monoclonal antibody AR711 has been explored as inhaled, self-administered therapy for mild to moderate COVID-19 patients to inhibit the receptor-binding domain (RBD) of the SARS-CoV2 virus and its constantly evolving variants [9]. Nanobodies (Nb) are antibody-like molecules discovered from the blood of camels [22]. Nanobodies $(\mathrm{Nb})$ have been shown to block the binding between the SARS- CoV-2-RBD variants to the ACE2 (angiotensin- converting enzyme 2) receptor. In 
particular, Nb11-59 can be a potent preventative and therapeutic agent against COVID- 19, especially through inhalation delivery [22]. Inhaled ciclesonide also shows strong antiviral actions against SARS-COV-2 and is promising to prevent virally induced lung injuries in the alveolar region [23, 24]. Inhalation (via Respimat ${ }^{\circledR}$ inhaler) of modified ACE2 has been evaluated as a decoy (that virus will bind with) to reduce viral infection of actual respiratory cells. Wu et al. [25] reported positive outcomes of the inhalation of freeze-dried plasminogen for fibrin degradation in 5 clinically moderate cases from reduced range and density in image opacity and improved oxygen saturation. Other inhalable candidates as antiviral agents include furosemide [26, 27], nebulized unfractionated heparin [28], Nanostructured lipid (salinomycin) [29], and freeze-dried Remdesivir [30]. All these new formulations are uniquely engineered to be delivered via inhalation and tackle the ailment directly in the lungs.

\section{Physiological Variation due to Corona Viral Infection}

\subsection{What has been learned from COVID-19 Patients?}

Coronaviruses can infect the cells of the entire respiratory tract, but the most severe damages occur in the lungs [31, 32]. COVID-19 patients often exhibit symptoms of acute respiratory distress. Common patterns of the lung physiological variations revealed by computed tomography (CT) include ground-glass opacification, bronchial wall thickening, lobular nodules, and vascular dilatation [33, 34]. Detailed pathological manifestations include hyaline membrane formation, white mucous, accumulation of gray-white viscous fluids, edema, lung consolidation, and lung hemorrhage [35-37]. Within the alveoli specifically, viral pathogenesis induces fibroblast and fibrin agglomeration, giant pneumocytes, lymphocytic inflammation, and infiltration of lymphocytes, macrophages, and neutrophils [38-40]. Small vessel thrombosis and pulmonary arterial cytoplasmic vacuolization have also been reported [41]. The diffuse alveolar damage (global injury) is also often observed in COVID-19 patients; these lung injuries can persist for a lifetime with different degrees of respiration impairments [42].

Autopsy reports showed the predominant histopathological modifications in the alveoli being microthrombi, capillary congestion, and intra-alveolar fibrin exudation resultant in disseminating alveolar damage and bronchopneumonia [43]. Another frequently observed histological damage pattern is the greater fibrotic evolution in the lungs of patients with mechanical ventilation [44]. Cases of pulmonary deposition of neutrophilic granulocytes were also reported, likely resulting from accompanying bacterial infections [45].

\subsection{Needs of Quantitative Lung Remodeling for Reliable Pulmonary Dosimetry}

Lung structural variations can have a marked impact on the delivery efficiency of inhaled therapeutic agents [46]. The endpoint of inhaled therapeutics is directly correlated to the delivered dose (and its distribution) in the lung, and particularly, the nominal doses on the target cells. The optimal dose depends on the location of the COVID-infected tissues, as well as the severity of the infection. If the drugs are delivered at a suboptimal rate or to a site away from the diseased tissue, the therapeutic efficacy can be remarkably compromised.

The prevailing cause of clinical deterioration and mortality is the cytokine storm in the pulmonary alveolar region [47]. Alveoli play a pivotal part during the inception and progression of immune responses to viral invasions, where Type-II cells are attacked, and gas exchange is compromised due to liquid filling. Studying pulmonary dosimetry in viral-remodeled lungs will improve our assessment and quantification of actual dosing to the diseased sites in COVID19 patients and thus develop more reliable dose-outcome profiles [48].

Up to date, reports of inhalation therapeutics in diseased lungs with disease-induced structural modifications are scant as opposed to the widely available reports on the effects of respiration, devices, aerosol properties, and even geometrical variations in the upper airways [49-58]. Even with tremendous progress in CT/MRI imaging, developing a lung model retaining the branches up to the ninth generation (G9) can still be highly challenging [59]. It will be even more demanding to evaluate the delivery variation in the disease-modified lungs, which often needs exceedingly highresolution CT images to capture fine morphological modifications, as well as a sufficient number of patient samples to reach a statistically significant conclusion. Therefore, most of the previous inhalation studies have only considered upper airway variants [60, 61], or a limited number of patient-specific lungs [51, 62-64]. Hurdled by potential radiation risks (CT), research ethics issues, and low clinical image resolutions, CT-based patient-specific lung geometry models beyond G9 are still scarce, with even fewer models of disease-modified lungs.

\subsection{Characterizing Lung Remodeling in COVID-19 Patients}

Figure 1a shows the 3D lungs based on CT scans of eight COVID-19 infected patients (4 males and four females, aged from 34-64 years old) using Slicer. The CT scans and patient information (age, gender, \& country of origin) were open to the medical community to accelerate COVID-related research and were sourced from the website covidctscans.org. 3D Slicer was used to segment healthy and diseased lungs, i.e., poorly aerated lungs (PAL), and non-aerated lungs (NAL), based on their different densities. Lanza et al. [65] reported using the Hounsfield unit (HU) intervals of $-500 \mathrm{HU}$ to $-100 \mathrm{HU}$ to isolate PAL, and $-100 \mathrm{HU}$ to $100 \mathrm{HU}$ to isolate NAL in their quantitative chest CT 
analysis of COVID-19 patients. By contrast, the healthy lungs were segmented using the threshold of -3024 HU to $500 \mathrm{HU}$. In order to quantitatively compare patients to each other, the volumes of each segment in each segmentation were calculated using the segment statistics module in 3D Slicer. The volume ratio of the PAL and NAL, as well as the total diseased lungs (PAL plus NAL = DIS), relative to the total lung volume, were calculated for each patient. Figure $1 \mathrm{~b}$ shows the average volume ratio and standard deviation of the PAL, NAL, and DIS based on the eight patients. Around $4 \%$ of the lungs $(\mathrm{SD}=3.5 \%)$ were poorly ventilated and $1.6 \%(\mathrm{SD}=1.5 \%)$ were not ventilated at all. The intersubject variability was also large (Figure 1b), indicating different severities of the COVID-19 infection among patients.

(a) COVID-infected lungs

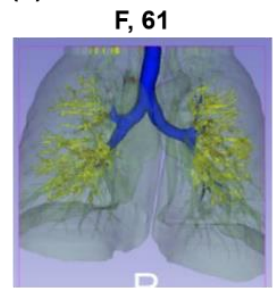

F, 64

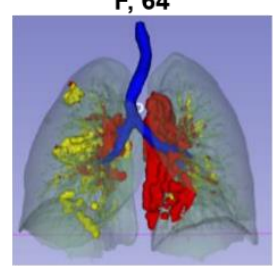

M, 47

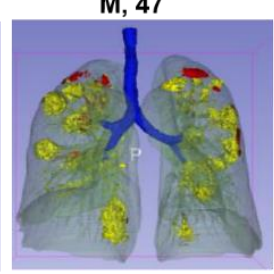

F, 55

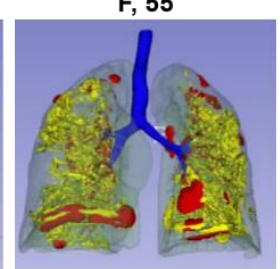

F, 41

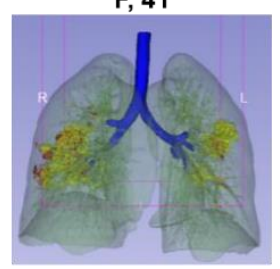

F, 34

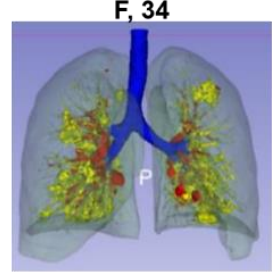

M, 32

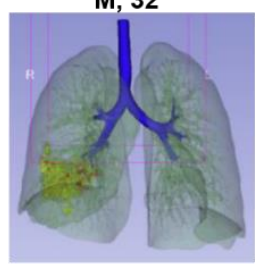

M, 50

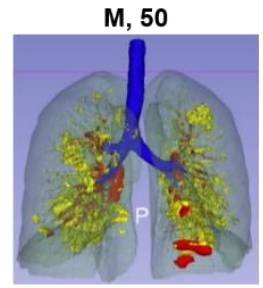

(b) Volume percentage

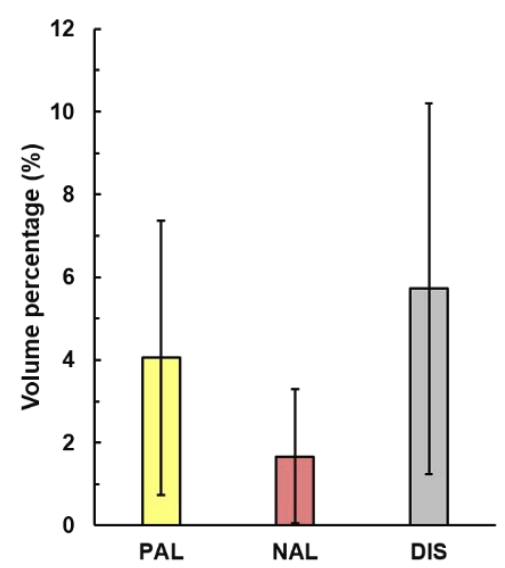

Figure 1. Average volume ratio and standard deviation for poorly aerated lungs (PAL, yellow), non-aerated lungs (NAL, red), and total diseased lungs (PAL \& NAL, gray)

The variability due to gender and age is shown in Figures $2 a$ and $2 b$, respectively. Based on this dataset, the female patients had a higher percentage of NAL and total diseased lung than males. Considering the age effect, the group between 50-60 years old is significantly higher than other age groups (Figure 2b). As aforementioned, the comparison was based on a limited dataset (eight patients) and our intent here was not to establish population-based statistics but to compare the compromised lung ratios in this dataset among different genders and age groups. A higher number of patients could also provide more information about the spectrum of variability in health and disease. In addition, other risk factors for the development of severe COVID-19 illness, such as ethnicity, obesity, and pre-existing conditions, could be investigated.

(a) Gender

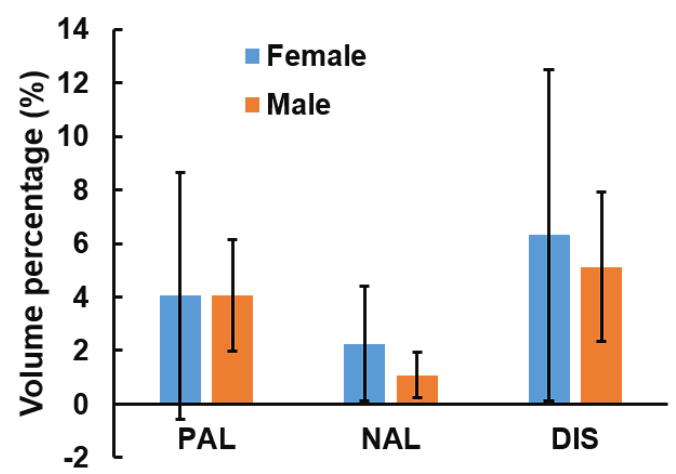

(b) Age

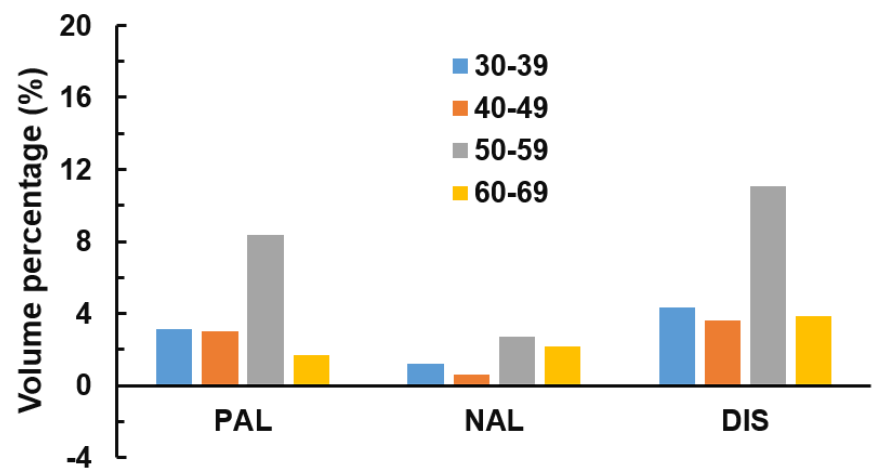

Figure 2. Volume ratio for poorly aerated lungs (PAL, yellow), non-aerated lungs (NAL, red), and total diseased lungs (PAL\& NAL, gray) vs. (a) gender, and (b) age

\section{Synergize CT-based Lung Generation and Algorithm-based Remodeling}

We have developed a new segmentation method that can construct patient-specific lung branches up to G13. By contrast, conventional segmentation methods can only capture good-quality branching geometry up to G6-8 unless with extremely high-resolution CT data $[66,67]$. Factors that contribute to this challenge include image artifacts, low resolution, and software segmentation algorithms. To address this challenge, a new segmentation method will be used that includes regional segmentation, manual fixing, and contrast enhancement, which has allowed us to reconstruct a 
lung branching geometry up to G13 (Figures 3a-e) based on a CT dataset that could otherwise be segmented to G7. Even though our new method is still labor-intensive and time-consuming, it can generate patient-specific lung geometries with peripheral (and smaller) bronchioles. Patient-specific lung volume geometries up to G13 are still scarce, and nearly all of them were reconstructed based on exceedingly high-resolution CT scans from specialized labs, which cannot be practically acquired in clinical settings [67]. In this sense, the new segmentation method can be critical to investigate the COVID-infected lungs considering that exceedingly high-resolution CT scans are often not an option to patients due to increased radiation risks. A patient-specific lung volume geometry that retains peripheral bronchioles up to G13 allows the analysis of flow details and aerosol dynamics in these small airways and thus provides a more accurate dosimetry estimation. By contrast, previous numerical studies of peripheral airways have often adopted oversimplified geometries and/or neglected upper stream flow effects, both of which can notably alter the inhaled particle behavior and fate [68, 69]. More importantly, due to such simplifications, the inter-subject variability and disease-associated effects cannot be reliably predicted.

(a) Segmentation

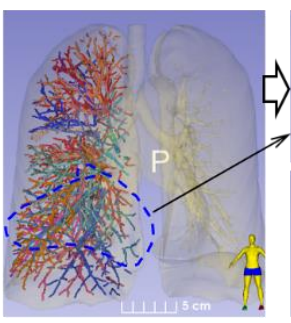

(b) Manual fixing

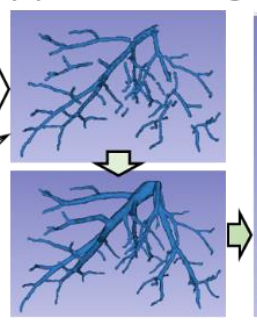

(c) Right lung

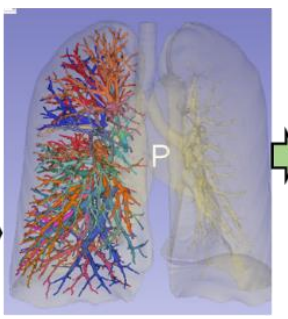

(d) Right lung: 3 lobes

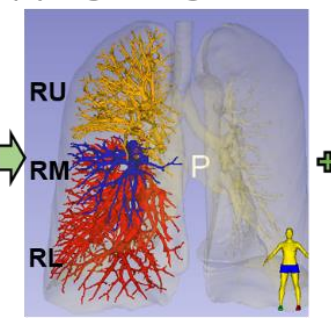

(e) Left lung

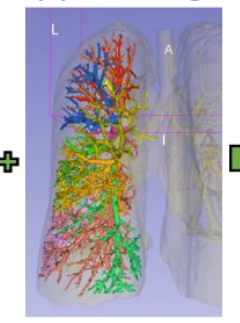

(f) Lung (G16)

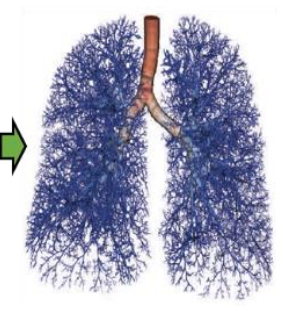

Figure 3. Patient-specific lung morphology reconstruction using Slicer: (a) bronchiole-wise segmentation, (b) manual fixing and smoothing, (c) a fixed right lung, (d) lobe separation, (e) left lung, and (f) a complete conducting lung geometry. The downstream generations were generated using the space-filling algorithm guided by the CT-based lobe volumes.

After developing the CT-based lung geometry, the downstream generations will be generated using the spacefilling algorithm guided by the CT-based lobe volumes (Figure 3f). Note that these downstream generations are only centerlines (or skeleton) and do not have a physical volume, which cannot be used directly for computational mesh generation and flow-particle simulations. The alveolar or acinar structures can be captured using micro-CT [70-72]. However, studies of airflows and aerosol dynamics in micro-CT-based alveolar models are still rare, with most of such studies being conducted in algorithm-based alveoli geometries [73-79].

(a) SSM-based remodeling (G9) Compliance (C)
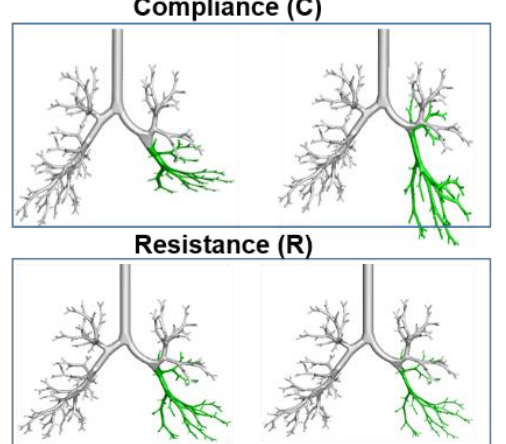

(b) Lung cast and remodeling (G12)

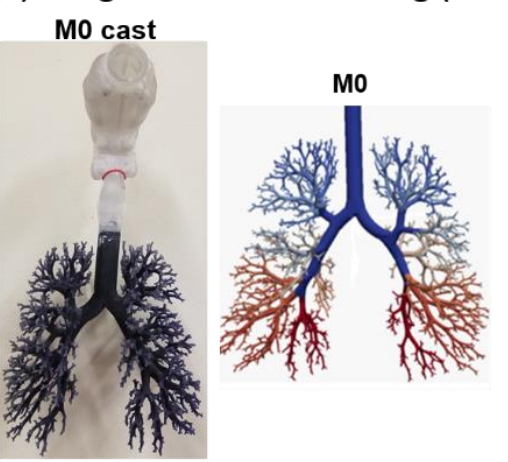

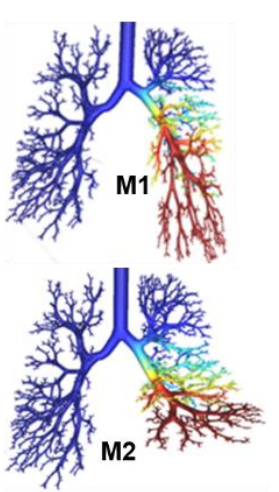

\section{(c) G16 lung}
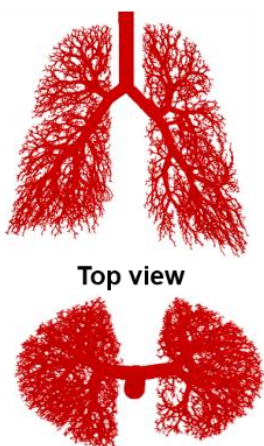

Figure 4. Modeling normal and diseased lungs using statistical shape modeling: (a) compliance- and resistance-related structural modification in the left lower lobe of a G9 lung, (b) lung casts and structural remodeling in a G12 lung, and (c) a space-filling lung volume model with the complete conducting airway (G0-G16).

To investigate inhalation therapeutics in disease-modified lungs, statistical shape modeling (SSM) has been explored in developing population-average shape and generating new "simulated" shape models [80]. This method was first developed as an imaging processing algorithm in computer graphics in the 1990s and has become popular in other disciplines like biomechanics [81, 82], biometrics [83], evolutional biology [84], anthropology [85], and forensics [86]. SSM uses the dataset comparing multiple shape models as a matrix and extracts the predominant features as leading eigenvectors, which will be further used in a linear combination to create new shape models [80]. A region of interest (ROI) can be delineated, and the shape modifications can be made in the ROI only. More desirably, the amplitude of shape modification can be controlled by varying the coefficient of the eigenvector in the linear combination [87]. This makes SSM well suited to examine the effects of lung structural modifications and the resultant variations in drug delivery [88]. In addition, an infinite number of new lung models can be generated by varying the eigenvector coefficients. 
The SSM has been successfully implemented to generate lung models with varying compliances and resistances (Figure 4a). A database consisting of 40 lung morphological models was used as the training set and the region of interest (ROI) was the left lower (LL) lobe. Models with different levels of caliber remodeling in the LL lobe were generated by adjusting the coefficients of the compliance- or constriction-associated eigenvectors [3]. Inhalation dosimetry in the airways with varying constriction levels has been numerically investigated for both orally [89] and nasally [90] inhaled nanoparticles.

Figure $4 \mathrm{~b}$ shows a lung (and a 3D printed cast) with 3,000 branches and extending to G12, which was generated using an in-house software Lung4CerE [91]. Lung4CerE can generate various kinds of 4D (= 3D space + time axis) models of the human bronchiolar-alveolar system [91]. The model format is in VTK (visualization toolkit) format, which can be converted to IGS, SAT, or STL format to conduct CFD computations [92]. The hollow cast (the second panel, Figure 4a) was fabricated using a ProJet 3D printer with a printing layer thickness of $16 \mu \mathrm{m}$, which captured the fine details of the lung cast. The right panel of Figure $4 \mathrm{~b}$ shows the compliance-related remodeling in the LL lobe of a G12 lung. Substantial discrepancies are expected in both the lobar ventilation and MDI droplet deposition on both the total, regional, and local basis.

Figure 4c shows a lung volume model up to G16 (red color). This model was generated using a specialized morphogenesis software GeoDict (Math2Market, Germany). Around 65,000 distal outlets were retained in this geometry. Due to the prohibitive requirement of computational resources, CFD simulations in this model are still impractical at this stage but can be implemented in the future to investigate pulmonary drug delivery in both normal and diseased lungs. This model can also serve as guidance in developing stochastic single-path whole lung models. One key question regarding the single-path whole lung models is how well can it represent the whole lung in characterizing the inhalation dosimetry of inhaled agents? Factors that support this question include: (1) the truncated bronchioles will affect the airflows in the retained single-path bronchioles, (2) the flow and particle dynamics can vary in different lobes due to gravity and streamline curvature, (3) the ventilation into the lower lobe is higher than the upper lobe. To answer this question, many single-path models leading to different positions of the lung periphery are needed. The centerlines of the model hereof can provide such paths, along which the bronchi and bronchioles can be reconstructed.

\section{Conclusion}

In this brief review, we summarized the recent developments of inhaled therapies (including vaccines). Inhaled therapies in COVID-19 patients can be more effective but have not yet been used in clinical practice. One key compounding factor in the inhaled therapy is the lung obstructions in COVID-19 patients, which can be highly heterogeneous in both anatomical location and severity. The synergy of CT-based patient-specific lung segmentation and algorithm-based airway remodeling is a promising tool to study pulmonary delivery in normal and diseased lungs and, as a consequence, to enhance the effect of pulmonary treatment and vaccine delivery in the future.

\section{Declarations}

\subsection{Author Contributions}

Conceptualization, J.X., X.S. and A.B.; methodology, J.X. and B.W.; software, B.W.; validation, J.X., B.W., X.S. and A.B.; formal analysis, J.X.; investigation, B.W. and X.S.; resources, J.X. and A.B.; data curation, J.X. and B.W.; writing - original draft preparation, J.X. and B.W.; writing-review and editing, J.X., X.S., B.W. and A.B.; visualization, J.X. and B.W.; supervision, J.X.; project administration, J.X. All authors have read and agreed to the published version of the manuscript.

\subsection{Funding}

The authors received no financial support for the research, authorship, and/or publication of this article.

\subsection{Acknowledgements}

Dr. Kitaoka Hiroko at Tokyo University of Agriculture and Technology, Japan was gratefully acknowledged for sharing the morphing module Lung4Cer.

\subsection{Ethical Approval}

The use of CT images from the open-source repository of COVID-19 CT scans http://covidctscans.org/ was approved by the UMass Lowell institutional review board (IRB).

\subsection{Data Availability Statement}

The data presented in this study are available on request from the corresponding author. The CT scans used in this study are publicly available through http://covidctscans.org/. 


\subsection{Conflict of Interest}

The authors declare that they have no known competing financial interests or personal relationships that could have appeared to influence the work reported in this paper.

\section{References}

[1] WHO (2021). World health orgnization coronavirus (COVID-19) dashboard: Available on: https://covid19.who.int/ (accessed on February 2021).

[2] Mitchell, J. P., Berlinski, A., Canisius, S., Cipolla, D., Dolovich, M. B., Gonda, I., ... Boushey, H. (2020). Urgent Appeal from International Society for Aerosols in Medicine (ISAM) During COVID-19: Clinical Decision Makers and Governmental Agencies Should Consider the Inhaled Route of Administration: A Statement from the ISAM Regulatory and Standardization Issues Networking Group. Journal of Aerosol Medicine and Pulmonary Drug Delivery, 33(4), $235-238$. doi:10.1089/jamp.2020.1622.

[3] Xi, J., Talaat, M., Si, X. A., \& Chandra, S. (2021). The application of statistical shape modeling for lung morphology in aerosol inhalation dosimetry. Journal of Aerosol Science, 151, 105623. doi:10.1016/j.jaerosci.2020.105623.

[4] Hussain, M., Renate, W.-H., Werner, H. (2011). Effect of intersubject variability of extrathoracic morphometry, lung airways dimensions and respiratory parameters on particle deposition. Journal of Thoracic Disease, 3(3), 156-170. doi:10.3978/j.issn.2072-1439.2011.04.03.

[5] Nayak, A. P., Deshpande, D. A., \& Penn, R. B. (2018). New targets for resolution of airway remodeling in obstructive lung diseases. F1000Research, 7, 680. doi:10.12688/f1000research.14581.1.

[6] Sköld, C. M. (2010). Remodeling in asthma and COPD - differences and similarities. The Clinical Respiratory Journal, 4, $20-27$. doi:10.1111/j.1752-699x.2010.00193.x.

[7] Staquicini, D. I., Barbu, E. M., Zemans, R. L., Dray, B. K., Staquicini, F. I., Dogra, P., Cardó-Vila, M., Miranti, C. K., Baze, W. B., Villa, L. L., Kalil, J., Sharma, G., Prossnitz, E. R., Wang, Z., Cristini, V., Sidman, R. L., Berman, A. R., Panettieri, R. A., Jr., Tuder, R. M., Pasqualini, R., Arap, W. (2021). Targeted phage display-based pulmonary vaccination in mice and non-human primates. Med, 2(3), 321-342.e328. doi:10.1016/j.medj.2020.10.005.

[8] Abdellatif, A. A. H., Tawfeek, H. M., Abdelfattah, A., El-Saber Batiha, G., Hetta, H. F. (2021). Recent updates in COVID-19 with emphasis on inhalation therapeutics: Nanostructured and targeting systems. J Drug Deliv Sci Technol, 63(102435. doi:10.1016/j.jddst.2021.102435.

[9] Piepenbrink, M. S., Park, J. G., Oladunni, F. S., Deshpande, A., Basu, M., Sarkar, S., Loos, A., Woo, J., Lovalenti, P., Sloan, D., Ye, C., Chiem, K., Bates, C. W., Burch, R. E., Erdmann, N. B., Goepfert, P. A., Truong, V. L., Walter, M. R., Martinez-Sobrido, L., Kobie, J. J. (2021). Therapeutic activity of an inhaled potent SARS-CoV-2 neutralizing human monoclonal antibody in hamsters. Cell Rep Med, 2(3), 100218. doi:10.1016/j.xcrm.2021.100218.

[10] Ramakrishnan, S., Nicolau, D. V., Langford, B., Mahdi, M., Jeffers, H., Mwasuku, C., ... Bafadhel, M. (2021). Inhaled budesonide in the treatment of early COVID-19 (STOIC): a phase 2, open-label, randomised controlled trial. The Lancet Respiratory Medicine. doi:10.1016/s2213-2600(21)00160-0.

[11] Rheault, T., Bengtsson, T., Rickard, K. (2020). Ensifentrine, a dual PDE3/PDE4 inhibitor, improves FEV1 regardless of smoking status or history of chronic bronchitis. Eur Respir J, 56(suppl 64), 4786. doi:10.1183/13993003.congress-2020.4786.

[12] Kobayashi, J., Murata, I. (2020). Nitric oxide inhalation as an interventional rescue therapy for COVID-19-induced acute respiratory distress syndrome. Ann Intensive Care, 10(1), 61. doi:10.1186/s13613-020-00681-9.

[13] Safaee Fakhr, B., Wiegand, S. B., Pinciroli, R., Gianni, S., Morais, C. C. A., Ikeda, T., ... Berra, L. (2020). High Concentrations of Nitric Oxide Inhalation Therapy in Pregnant Patients with Severe Coronavirus Disease 2019 (COVID-19). Obstetrics \& Gynecology, 136(6), 1109-1113. doi:10.1097/aog.0000000000004128.

[14] Halpin, D. M. G., Singh, D., Hadfield, R. M. (2020). Inhaled corticosteroids and COVID-19: a systematic review and clinical perspective. Eur Respir J, 55(2001009. doi:10.1183/13993003.01009-2020.

[15] Singh, D., Halpin, D. M. G. (2020). Inhaled corticosteroids and COVID-19-related mortality: confounding or clarifying? Lancet Respir Med, 8(11), 1065-1066. doi:10.1016/S2213-2600(20)30447-1.

[16] Haren, F. M. P., Richardson, A., Yoon, H., Artigas, A., Laffey, J. G., Dixon, B., ... Page, C. (2021). INHALEd nebulised unfractionated HEParin for the treatment of hospitalised patients with COVID-19 (INHALE-HEP): Protocol and statistical analysis plan for an investigator - initiated international metatrial of randomised studies. British Journal of Clinical Pharmacology. doi:10.1111/bcp.14714

[17] Mary, A., Hénaut, L., Macq, P. Y., Badoux, L., Cappe, A., Porée, T., Eckes, M., Dupont, H., Brazier, M. (2020). Rationale for COVID-19 treatment by nebulized interferon- $\beta-1 b$-Literature review and personal preliminary experience. Front Pharmacol, 11(1885). doi:10.3389/fphar.2020.592543. 
[18] Albariqi, A. H., Chang, R. Y. K., Tai, W., Ke, W.-R., Y.T, M., Chow, P. T., Kwok, P. C. L., Chan, H.-K. (2021). Inhalable hydroxychloroquine powders for potential treatment of COVID-19. J Aerosol Med Pulm Drug Deliv, 34(1), 20-31. doi:10.1089/jamp.2020.1648.

[19] Ferrer, G., Betancourt, A., Go, C. C., Vazquez, H., Westover, J. B., Cagno, V., Tapparel, C., Sanchez-Gonzalez, M. A. (2020). A nasal spray solution of grapefruit seed extract plus Xylitol displays virucidal activity against SARS-Cov-2 in vitro. BioRxiv, Preprint, 394114. doi:10.1101/2020.11.23.394114.

[20] Abdellatif, A. A. H. (2020). A plausible way for excretion of metal nanoparticles via active targeting. Drug Dev Ind Pharm, 46(5), 744-750. doi:10.1080/03639045.2020.1752710.

[21] Abdellatif, A. A. H., Ibrahim, M. A., Amin, M. A., Maswadeh, H., Alwehaibi, M. N., Al-Harbi, S. N., Alharbi, Z. A., Mohammed, H. A., Mehany, A. B. M., Saleem, I. (2020). Cetuximab conjugated with octreotide and entrapped calcium Alginate-beads for targeting Somatostatin Receptors. Sci Rep, 10(1), 4736. doi:10.1038/s41598-020-61605-y.

[22] Gai, J., Ma, L., Li, G., Zhu, M., Qiao, P., Li, X., Zhang, H., Zhang, Y., Chen, Y., Ji, W., Zhang, H., Cao, H., Li, X., Gong, R., Wan, Y. (2021). A potent neutralizing nanobody against SARS-CoV-2 with inhaled delivery potential. MedComm, 2(1), 101113. doi:10.1002/mco2.60.

[23] Iwabuchi, K., Yoshie, K., Kurakami, Y., Takahashi, K., Kato, Y., Morishima, T. (2020). Therapeutic potential of ciclesonide inahalation for COVID-19 pneumonia: Report of three cases. J Infect Chemother, 26(6), 625-632. doi:10.1016/j.jiac.2020.04.007.

[24] Matsuyama, S., Kawase, M., Nao, N., Shirato, K., Ujike, M., Kamitani, W., ... Fukushi, S. (2020). The Inhaled Steroid Ciclesonide Blocks SARS-CoV-2 RNA Replication by Targeting the Viral Replication-Transcription Complex in Cultured Cells. Journal of Virology, 95(1). doi:10.1128/jvi.01648-20.

[25] Wu, Y., Wang, T., Guo, C., Zhang, D., Ge, X., Huang, Z., Zhou, X., Li, Y., Peng, Q., Li, J. (2020). Plasminogen improves lung lesions and hypoxemia in patients with COVID-19. QJM: Int J Med, 113(8), 539-545. doi:10.1093/qjmed/hcaa121.

[26] Wang, Z., Wang, Y., Vilekar, P., Yang, S. P., Gupta, M., Oh, M. I., Meek, A., Doyle, L., Villar, L., Brennecke, A., Liyanage, I., Reed, M., Barden, C., Weaver, D. F. (2020). Small molecule therapeutics for COVID-19: repurposing of inhaled furosemide. PeerJ, 8(e9533. doi:10.7717/peerj.9533.

[27] Brennecke, A., Villar, L., Wang, Z., Doyle, L. M., Meek, A., Reed, M., ... Weaver, D. F. (2020). Is Inhaled Furosemide a Potential Therapeutic for COVID-19? The American Journal of the Medical Sciences, 360(3), $216-221$. doi:10.1016/j.amjms.2020.05.044.

[28] van Haren, F. M. P., Page, C., Laffey, J. G., Artigas, A., Camprubi-Rimblas, M., Nunes, Q., Smith, R., Shute, J., Carroll, M., Tree, J., Carroll, M., Singh, D., Wilkinson, T., Dixon, B. (2020). Nebulised heparin as a treatment for COVID-19: scientific rationale and a call for randomised evidence. Crit Care, 24(1), 454. doi:10.1186/s13054-020-03148-2.

[29] Pindiprolu, S., Kumar, C. S. P., Kumar Golla, V. S., P, L., K, S. C., S, K. E., R, K. R. (2020). Pulmonary delivery of nanostructured lipid carriers for effective repurposing of salinomycin as an antiviral agent. Med Hypotheses, 143(109858. doi:10.1016/j.mehy.2020.109858.

[30] Sahakijpijarn, S., Moon, C., Koleng, J. J., Christensen, D. J., Williams Iii, R. O. (2020). Development of Remdesivir as a Dry Powder for Inhalation by Thin Film Freezing. Pharmaceutics, 12(11). doi:10.3390/pharmaceutics12111002.

[31] Dimbath, E., Maddipati, V., Stahl, J., Sewell, K., Domire, Z., George, S., Vahdati, A. (2021). Implications of microscale lung damage for COVID-19 pulmonary ventilation dynamics: A narrative review. Life Sci, $274(119341$. doi:10.1016/j.lfs.2021.119341.

[32] Mohanty, S. K., Satapathy, A., Naidu, M. M., Mukhopadhyay, S., Sharma, S., Barton, L. M., Stroberg, E., Duval, E. J., Pradhan, D., Tzankov, A., Parwani, A. V. (2020). Severe acute respiratory syndrome coronavirus-2 (SARS-CoV-2) and coronavirus disease 19 (COVID-19) - anatomic pathology perspective on current knowledge. Diagn Pathol, $15(1), 103$. doi:10.1186/s13000-020-01017-8.

[33] Fernández-Pérez, G. C., Oñate Miranda, M., Fernández-Rodríguez, P., Velasco Casares, M., Corral de la Calle, M., Franco López, Á., ... Oñate Cuchat, J. M. (2021). SARS-CoV-2: what it is, how it acts, and how it manifests in imaging studies. Radiología (English Edition), 63(2), 115-126. doi:10.1016/j.rxeng.2020.10.006.

[34] Kim, E. A., Lee, K. S., Primack, S. L., Yoon, H. K., Byun, H. S., Kim, T. S., Suh, G. Y., Kwon, O. J., Han, J. (2002). Viral pneumonias in adults: radiologic and pathologic findings. Radiographics, 22 Spec No(S137-149. doi:10.1148/radiographics.22.suppl_1.g02oc15s137.

[35] Hanley, B., Lucas, S. B., Youd, E., Swift, B., Osborn, M. (2020). Autopsy in suspected COVID-19 cases. J Clin Pathol, 73(5), 239-242. doi:10.1136/jclinpath-2020-206522. 
[36] Zhou, B., Zhao, W., Feng, R., Zhang, X., Li, X., Zhou, Y., Peng, L., Li, Y., Zhang, J., Luo, J., Li, L., Wu, J., Yang, C., Wang, M., Zhao, Y., Wang, K., Yu, H., Peng, Q., Jiang, N. (2020). The pathological autopsy of coronavirus disease 2019 (COVID2019) in China: a review. Pathog Dis., 78(3). doi:10.1093/femspd/ftaa026.

[37] Calabrese, F., Pezzuto, F., Fortarezza, F., Hofman, P., Kern, I., Panizo, A., von der Thüsen, J., Timofeev, S., Gorkiewicz, G., Lunardi, F. (2020). Pulmonary pathology and COVID-19: lessons from autopsy. The experience of European Pulmonary Pathologists. Virchows Archiv: Eur J Pathol, 477(3), 359-372. doi:10.1007/s00428-020-02886-6.

[38] Santurro, A., Scopetti, M., D'Errico, S., Fineschi, V. (2020). A technical report from the Italian SARS-CoV-2 outbreak. Postmortem sampling and autopsy investigation in cases of suspected or probable COVID-19. Forensic Sci Med Pathol, 16(3), 471-476. doi:10.1007/s12024-020-00258-9.

[39] Tian, S., Hu, W., Niu, L., Liu, H., Xu, H., \& Xiao, S.-Y. (2020). Pulmonary Pathology of Early-Phase 2019 Novel Coronavirus (COVID-19) Pneumonia in Two Patients with Lung Cancer. Journal of Thoracic Oncology, 15(5), $700-704$. doi:10.1016/j.jtho.2020.02.010

[40] Yao, X. H., He, Z. C., Li, T. Y., Zhang, H. R., Wang, Y., Mou, H., Guo, Q., Yu, S. C., Ding, Y., Liu, X., Ping, Y. F., Bian, X. W. (2020). Pathological evidence for residual SARS-CoV-2 in pulmonary tissues of a ready-for-discharge patient. Cell Res, 30(6), 541-543. doi:10.1038/s41422-020-0318-5.

[41] Copin, M. C., Parmentier, E., Duburcq, T., Poissy, J., Mathieu, D. (2020). Time to consider histologic pattern of lung injury to treat critically ill patients with COVID-19 infection. Intensive Care Med, 46(6), 1124-1126. doi:10.1007/s00134-020-06057-8.

[42] Batah, S. S., Fabro, A. T. (2021). Pulmonary pathology of ARDS in COVID-19: A pathological review for clinicians. Respir Med, 176(106239. doi:10.1016/j.rmed.2020.106239.

[43] Menter, T., Haslbauer, J. D., Nienhold, R., Savic, S., Hopfer, H., Deigendesch, N., Frank, S., Turek, D., Willi, N., Pargger, H., Bassetti, S., Leuppi, J. D., Cathomas, G., Tolnay, M., Mertz, K. D., Tzankov, A. (2020). Postmortem examination of COVID19 patients reveals diffuse alveolar damage with severe capillary congestion and variegated findings in lungs and other organs suggesting vascular dysfunction. Histopathology, 77(2), 198-209. doi:10.1111/his.14134.

[44] Barisione, E., Grillo, F., Ball, L., Bianchi, R., Grosso, M., Morbini, P., Pelosi, P., Patroniti, N. A., De Lucia, A., Orengo, G., Gratarola, A., Verda, M., Cittadini, G., Mastracci, L., Fiocca, R. (2021). Fibrotic progression and radiologic correlation in matched lung samples from COVID-19 post-mortems. Virchows Archiv : an international journal of pathology, 478(3), 471485. doi:10.1007/s00428-020-02934-1.

[45] Bourgonje, A. R., Abdulle, A. E., Timens, W., Hillebrands, J. L., Navis, G. J., Gordijn, S. J., Bolling, M. C., Dijkstra, G., Voors, A. A., Osterhaus, A. D., van der Voort, P. H., Mulder, D. J., van Goor, H. (2020). Angiotensin-converting enzyme 2 (ACE2), SARS-CoV-2 and the pathophysiology of coronavirus disease 2019 (COVID-19). J Pathol, 251(3), 228-248. doi:10.1002/path.5471.

[46] Bauer, C., Krueger, M., Lamm, W. J. E., Glenny, R. W., Beichel, R. R. (2020). lapdMouse: associating lung anatomy with local particle deposition in mice. J Appl Physiol, 128(2), 309-323. doi:10.1152/japplphysiol.00615.2019.

[47] Soy, M., Keser, G., Atagündüz, P., Tabak, F., Atagündüz, I., Kayhan, S. (2020). Cytokine storm in COVID-19: pathogenesis and overview of anti-inflammatory agents used in treatment. Clin Rheumatol, 39(7), 2085-2094. doi:10.1007/s10067-02005190-5.

[48] Yuan, J., Chiofolo, C. M., Czerwin, B. J., Karamolegkos, N., Chbat, N. W. (2021). Alveolar tissue fiber and surfactant effects on lung mechanics - model development and validation on ARDS and IPF patients. IEEE Open J Eng Med, 2(44-54. doi:10.1109/OJEMB.2021.3053841.

[49] De Backer, J. W., Vos, W. G., Burnell, P., Verhulst, S. L., Salmon, P., De Clerck, N., De Backer, W. (2009). Study of the variability in upper and lower airway morphology in Sprague-Dawley rats using modern micro-CT scan-based segmentation techniques. Anat Rec, 292(5), 720-727. doi:10.1002/ar.20877.

[50] Hofmann, W., Asgharian, B., Winkler-Heil, R. (2002). Modeling intersubject variability of particle deposition in human lungs. J Aerosol Sci, 33(219-235. doi:10.1016/S0021-8502(01)00167-7.

[51] Schroeter, J. D., Garcia, G. J. M., Kimbell, J. S. (2010). A computational fluid dynamics approach to assess interhuman variability in hydrogen sulfide nasal dosimetry. Inhal Toxicol, 22(4), 277-286. doi:10.3109/08958370903278077.

[52] Thekedar, B., Oeh, U., Szymczak, W., Hoeschen, C., Paretzke, H. G. (2011). Influences of mixed expiratory sampling parameters on exhaled volatile organic compound concentrations. J Breath Res, 5(1). doi:10.1088/1752-7155/5/1/016001.

[53] Vijverberg, S. J. H., Koenderman, L., Koster, E. S., van der Ent, C. K., Raaijmakers, J. A. M., Maitland-van der Zee, A. H. (2011). Biomarkers of therapy responsiveness in asthma: pitfalls and promises. Clin Exp Allergy, 41(5), 615-629. doi:10.1111/j.1365-2222.2011.03694.x.

[54] Xi, J., Kim, J., Si, X. A., \& Zhou, Y. (2013). Diagnosing obstructive respiratory diseases using exhaled aerosol fingerprints: A feasibility study. Journal of Aerosol Science, 64, 24-36. doi:10.1016/j.jaerosci.2013.06.003 
[55] Xi, J., Yang, T. (2019). Variability in oropharyngeal airflow and aerosol deposition due to changing tongue positions. J Drug Deliv Sci Technol, 49(674-682. doi:10.1016/j.jddst.2019.01.006.

[56] Xi, J., Si, X. A., Kim, J. W., (2015). Characterizing respiratory airflow and aerosol condensational growth in children and adults using an imaging-CFD approach, in: Heat Transfer and Fluid Flow in Biological Processes, Academic Press, pp. 125155. doi:10.1016/B978-0-12-408077-5.00005-5.

[57] Xi, J., Kim, J., Si, X. A. (2016). Effects of nostril orientation on airflow dynamics, heat exchange, and particle depositions in human noses. Eur J Mech B Fluids, 55(215-228. doi:10.1016/j.euromechflu.2015.08.014.

[58] Si, X., Xi, J., Kim, J. (2013). Effect of laryngopharyngeal anatomy on expiratory airflow and submicrometer particle deposition in human extrathoracic airways. Open J Fluid Dyn, 3(4), 286-301. doi:10.4236/ojfd.2013.34036.

[59] Xi, J., Longest, P. W. (2008). Evaluation of a drift flux model for simulating submicrometer aerosol dynamics in human upper tracheobronchial airways. Ann Biomed Eng, 36(10), 1714-1734. doi:10.1007/s10439-008-9552-6.

[60] Xi, J., Zhang, Z., Si, X. (2015). Improving intranasal delivery of neurological nanomedicine to the olfactory region using magnetophoretic guidance of microsphere carriers. Int J Nanomedicine, 10(1211-1222. doi:10.2147/IJN.S77520.

[61] Xi, J., Si, X. A., Peters, S., Nevorski, D., Wen, T., Lehman, M. (2017). Understanding the mechanisms underlying pulsating aerosol delivery to the maxillary sinus: In vitro tests and computational simulations. Int $\mathrm{J}$ Pharm, 520(1-2), $254-266$. doi:10.1016/j.ijpharm.2017.02.017.

[62] Garcia, G. J. M., Schroeter, J. D., Segal, R. A., Stanek, J., Foureman, G. L., Kimbell, J. S. (2009). Dosimetry of nasal uptake of water-soluble and reactive gases: A first study of interhuman variability. Inhal Toxicol, 21(5-7), 607-618. doi:10.1080/08958370802320186.

[63] Xi, J., Berlinski, A., Zhou, Y., Greenberg, B., Ou, X. (2012). Breathing resistance and ultrafine particle deposition in nasallaryngeal airways of a newborn, an infant, a child, and an adult. Ann Biomed Eng, 40(12), 2579-2595. doi:10.1007/s10439012-0603-7.

[64] Segal, R. A., Kepler, G. M., Kimbell, J. S. (2008). Effects of differences in nasal anatomy on airflow distribution: A comparison of four individuals at rest. Ann Biomed Eng, 36(11), 1870-1882. doi:10.1007/s10439-008-9556-2.

[65] Lanza, E., Muglia, R., Bolengo, I., Santonocito, O. G., Lisi, C., Angelotti, G., Morandini, P., Savevski, V., Politi, L. S., Balzarini, L. (2020). Quantitative chest CT analysis in COVID-19 to predict the need for oxygenation support and intubation. Eur Radiol, 30(12), 6770-6778. doi:10.1007/s00330-020-07013-2.

[66] Xi, J., Longest, P. W. (2007). Transport and deposition of micro-aerosols in realistic and simplified models of the oral airway. Ann Biomed Eng, 35(4), 560-581. doi:10.1007/s10439-006-9245-y.

[67] Corley, R. A., Kabilan, S., Kuprat, A. P., Carson, J. P., Minard, K. R., Jacob, R. E., Timchalk, C., Glenny, R., Pipavath, S., Cox, T., Wallis, C. D., Larson, R. F., Fanucchi, M. V., Postlethwait, E. M., Einstein, D. R. (2012). Comparative computational modeling of airflows and vapor dosimetry in the respiratory tracts of rat, monkey, and human. Toxicol Sci, 128(2), 500-516. doi:10.1093/toxsci/kfs168.

[68] Longest, P. W., Vinchurkar, S. (2007). Validating CFD predictions of respiratory aerosol deposition: effects of upstream transition and turbulence. J Biomech, 40(305-316. doi:10.1016/j.jbiomech.2006.01.006.

[69] Xi, J., Longest, P. W., Martonen, T. B. (2008). Effects of the laryngeal jet on nano- and microparticle transport and deposition in an approximate model of the upper tracheobronchial airways. J Appl Physiol, 104(6), 1761-1777. doi:10.1152/japplphysiol.01233.2007.

[70] Watz, H., Breithecker, A., Rau, W. S., Kriete, A. (2005). Micro-CT of the human lung: imaging of alveoli and virtual endoscopy of an alveolar duct in a normal lung and in a lung with centrilobular emphysema--initial observations. Radiology, 236(3), 1053-1058. doi:10.1148/radiol.2363041142.

[71] Parameswaran, H., Bartolák-Suki, E., Hamakawa, H., Majumdar, A., Allen, P. G., Suki, B. (2009). Three-dimensional measurement of alveolar airspace volumes in normal and emphysematous lungs using micro-CT. J Appl Physiol, 107(2), 583592. doi:10.1152/japplphysiol.91227.2008.

[72] Hochhegger, B., Langer, F. W., Irion, K., Souza, A., Moreira, J., Baldisserotto, M., Pallaoro, Y., Muller, E., Medeiros, T. M., Altmayer, S., Marchiori, E. (2019). Pulmonary acinus: understanding the computed tomography findings from an acinar perspective. Lung, 197(3), 259-265. doi:10.1007/s00408-019-00214-7.

[73] Tippe, A., Tsuda, A. (2000). Recirculating flow in an expanding alveolar model: Experimental evidence of flow-induced mixing of aerosols in the pulmonary acinus. J Aerosol Sci, 31(8), 979-986. doi:10.1016/S0021-8502(99)00572-8.

[74] Kitaoka, H., Tamura, S., Takaki, R. (2000). A three-dimensional model of the human pulmonary acinus. J Appl Physiol, 88(6), 2260-2268. doi:10.1152/jappl.2000.88.6.2260. 
[75] Darquenne, C., Paiva, N. (1996). Two- and three-dimensional simulations of aerosol transport and deposition in alveolar zone of human lung. J Appl Physiol, 80(4), 1401-1414. doi:10.1152/jappl.1996.80.4.1401.

[76] Hofemeier, P., Koshiyama, K., Wada, S., Sznitman, J. (2018). One (sub-)acinus for all: Fate of inhaled aerosols in heterogeneous pulmonary acinar structures. Eur J Pharm Sci, 113(53-63. doi:10.1016/j.ejps.2017.09.033.

[77] Talaat, M., Si, X. A., Kitaoka, H., Xi, J. (2021). Septal destruction enhances chaotic mixing and increases cellular doses of nanoparticles in emphysematous acinus. Nano Express, 2(1), 010015. doi:10.1088/2632-959x/abe0f8.

[78] Talaat, M., Si, X. A., Tanbour, H., Xi, J. (2019). Numerical studies of nanoparticle transport and deposition in terminal alveolar models with varying complexities. Med One, 4(5), e190018. doi:10.20900/mo.20190018.

[79] Xi, J., Talaat, M. (2019). Nanoparticle deposition in rhythmically moving acinar models with iteralveolar septal apertures. Nanomaterials, 9(8). doi:10.3390/nano9081126.

[80] Chandra, S. S., Xia, Y., Engstrom, C., Crozier, S., Schwarz, R., Fripp, J. (2014). Focused shape models for hip joint segmentation in 3D magnetic resonance images. Med Image Anal, 18(3), 567-578. doi:10.1016/j.media.2014.02.002.

[81] Castro-Mateos, I., Pozo, J. M., Cootes, T. F., Wilkinson, J. M., Eastell, R., Frangi, A. F. (2014). Statistical shape and appearance models in osteoporosis. Curr Osteoporos Rep, 12(2), 163-173. doi:10.1007/s11914-014-0206-3.

[82] Fitzpatrick, C., FitzPatrick, D., Lee, J., Auger, D. (2007). Statistical design of unicompartmental tibial implants and comparison with current devices. Knee, 14(2), 138-144. doi:http://dx.doi.org/10.1016/j.knee.2006.11.005.

[83] Quan, W., Matuszewski, B. J., Shark, L.-K. (2016). Statistical shape modelling for expression-invariant face analysis and recognition. Pattern Anal Appl, 19(3), 765-781. doi:10.1007/s10044-014-0439-x.

[84] Macdonald-Wallis, C. (2014). Statistical Analysis of Human Growth and Development. International Journal of Epidemiology, 43(2), 635-636. doi:10.1093/ije/dyu068.

[85] Krishan, K., Chatterjee, P. M., Kanchan, T., Kaur, S., Baryah, N., Singh, R. K. (2016). A review of sex estimation techniques during examination of skeletal remains in forensic anthropology casework. Forensic Sci Int, 261(165.e161-168. doi:10.1016/j.forsciint.2016.02.007.

[86] Hauser, R., Smolinski, J., Gos, T. (2005). The estimation of stature on the basis of measurements of the femur. Forensic Sci Int, 147(2-3), 185-190. doi:10.1016/j.forsciint.2004.09.070.

[87] Jakab, A., Blanc, R., Berenyi, E. L., Szekely, G. (2012). Generation of individualized thalamus target maps by using statistical shape models and thalamocortical tractography. Am J Neuroradiol, 33(11), 2110-2116. doi:10.3174/ajnr.A3140.

[88] Xi, J., Zhao, W. (2019). Correlating exhaled aerosol images to small airway obstructive diseases: A study with dynamic mode decomposition and machine learning. PloS ONE, 14(1). doi:10.1371/journal.pone.0211413.

[89] Talaat, M., Si, X. A., Dong, H., Xi, J. (2021). Leveraging statistical shape modeling in computational respiratory dynamics: nanomedicine delivery in remodeled airways. Comput Meth Prog Biomed, 204(106079. doi:10.1016/j.cmpb.2021.106079.

[90] Si, X. A., Talaat, M., Su, W. C., Xi, J. (2021). Inhalation dosimetry of nasally inhaled respiratory aerosols in the human respiratory tract with locally remodeled conducting lungs. Inhal Toxicol, in press(1-17. doi:10.1080/08958378.2021.1912860.

[91] Kitaoka, H., Nieman, G. F., Fujino, Y., Carney, D., DiRocco, J., Kawase, I. (2007). A 4-dimensional model of the alveolar structure. J Physiol Sci, 57(3), 175-185. doi:10.2170/physiolsci.RP000807.

[92] Kitaoka, H. (2011). The origin of frequency dependence of respiratory resistance: An airflow simulation study using a 4D pulmonary lobule model. Respirology, 16(3), 517-522. doi:10.1111/j.1440-1843.2011.01925.x. 